NISSUNA UMANA INVESTIGAZIONE SI PUO DIMANDARE VERA SCIENZIA S'ESSA NON PASSA PER LE MATEMATICHE DIMOSTRAZIONI LEONARDO DA VINCI

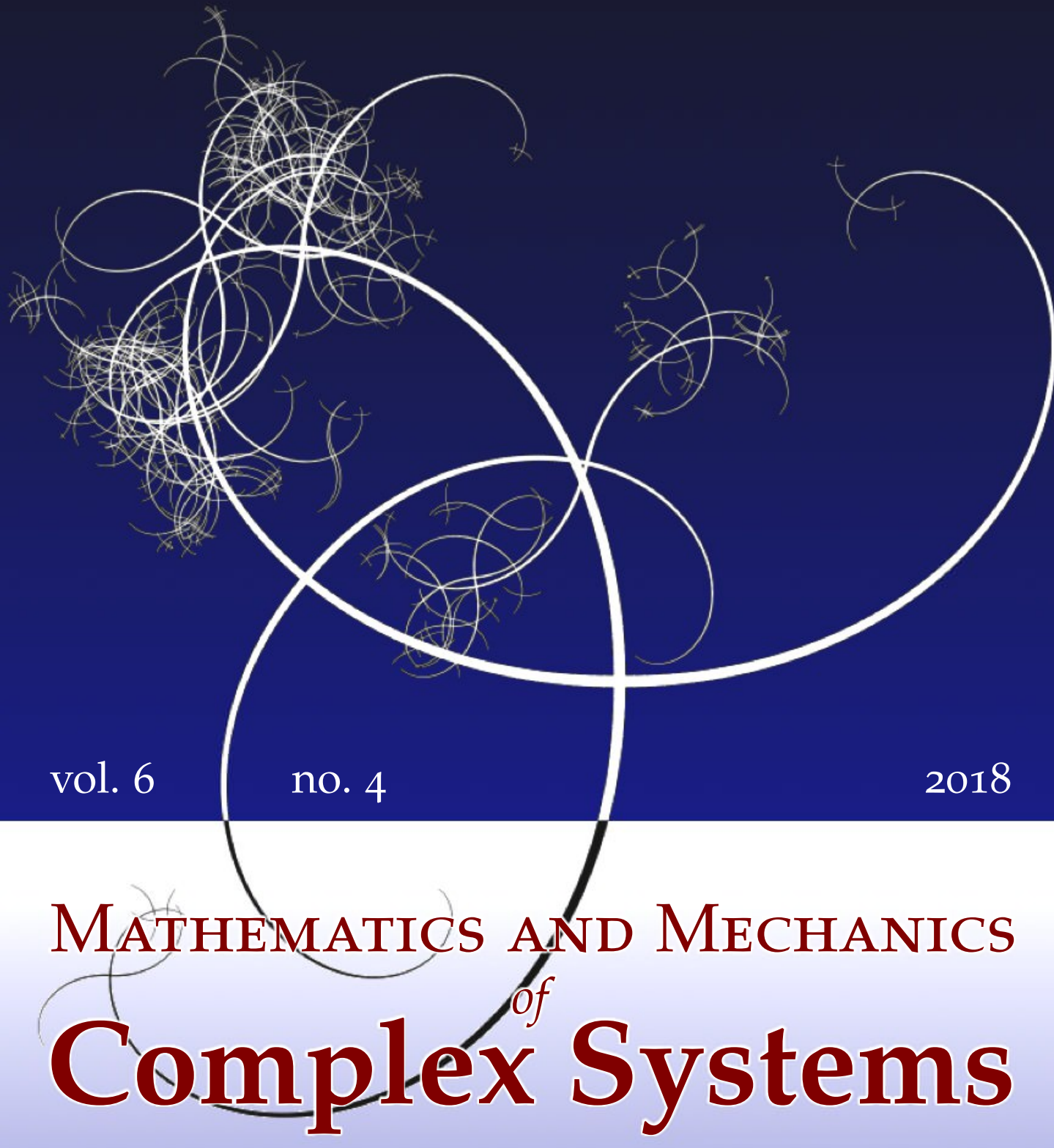

NARINDRA RANAIVOMIARANA, FRANÇOIS-XAVIER IRISARRI, DIMITRI BETTEBGHOR AND BORIS DESMORAT OPTIMAL ORTHOTROPY AND DENSITY DISTRIBUTION OF TWO-DIMENSIONAL STRUCTURES 


\title{
OPTIMAL ORTHOTROPY AND DENSITY DISTRIBUTION OF TWO-DIMENSIONAL STRUCTURES
}

\author{
NARINDRA RANAIVOMIARANA, FRANÇOIS-XAVIER IRISARRI, \\ DIMITRI BETTEBGHOR AND BORIS DESMORAT
}

\begin{abstract}
This paper describes an optimization methodology giving simultaneously the optimal spatial material distribution and the optimal material orthotropy distribution in a two-dimensional space. The spatial material distribution is parametrized by a density variable that defines the presence or absence of material. A general orthotropic material is parametrized by the polar invariants of the elasticity tensor. The criterion is the compliance that measures the global structural stiffness. The numerical procedure iterates successively between local minimizations and finite element calculations. Thanks to the polar method, the local minimizations are solved explicitly providing analytical solutions. An optimization of a beam shows the effectiveness of the method in finding concurrently the optimal shape and the optimal material.
\end{abstract}

\section{Introduction}

Reducing cost and weight of structures is a permanent challenge for the aeronautics industry. In this scope, topology optimization is used for the mass minimization problem [Allaire and Delgado 2016]. It gives an ideal repartition of material considering, for instance, global stiffness or eigenfrequency of a structure under loads and boundary conditions. The optimal shape or layout of the structure is then obtained. Topology optimization is widely used for isotropic materials [Bendsøe and Sigmund 2003; Sigmund and Maute 2013] such as metallic ones for example, but it does not optimize the material behavior, e.g., the anisotropy. The mass of the structure can also be reduced by optimizing the material that composes it. Composite structure optimization [Ghiasi et al. 2009; 2010; Sørensen and Lund 2013; Peeters et al. 2015] is used to design the material at each point of the structure. For instance, the optimal layup of laminates is sought by changing the orientations of plies, the thickness, or the stacking sequence with heuristic [Irisarri et al. 2009] or

\section{Communicated by Francesco dell'Isola.}

The authors acknowledge STELIA Aerospace for funding this project.

MSC2010: 74P10.

Keywords: topology optimization, SIMP, polar method, distributed orthotropy, material design. 
gradient-based methods [Sørensen and Lund 2013]. The composite optimization is generally done with a predefined shape of structure. Thus, topology optimization gives an optimal distribution of material [Rojas-Labanda and Stolpe 2015] without considering its optimal anisotropy and composite structure optimization [Ghiasi et al. 2009; 2010; Sørensen and Lund 2013; Peeters et al. 2015] gives an optimal anisotropy of the material without considering the optimal shape of the structure. Nonetheless, the shape and the material of the structure are closely related. To obtain an ideal structure, it is necessary to optimize the structure by considering the optimal spatial material distribution and the optimal material anisotropy distribution at the same time.

Rion and Bruyneel [2007] treat topology optimization of orthotropic material by considering fiber orientations in the optimization. The determination of the boundaries of the structure combined with that of optimal fiber path is treated in [Peeters et al. 2015], where the stiffness is parametrized by lamination parameters. Allaire and Delgado [2016] optimize laminated composite plates where the shape of each layer is determined concurrently with the stacking sequence. In this paper, we present an optimization methodology giving simultaneously the optimal shape and the optimal orthotropy distribution of the structure. The optimization is made on a general homogenized orthotropic material.

Parametrization of the shape and the anisotropy is necessary. First we choose the density method to parametrize the shape of the structure. The density variable determines at each point of the structure whether there is material or a void. The anisotropy of the material is characterized by its elasticity tensor. As we work on a general orthotropic material, we consider the homogenized elasticity tensor defined in a thermodynamically admissible domain. The elasticity tensor can be described by nine Cartesian coefficients. Since the material orthotropy varies through the structure, one should define a general frame to express the elasticity tensor. However, the use of Cartesian representation is cumbersome when changing frame. The polar method, introduced by Verchery [1982], uses invariants by change of frame to describe the elasticity tensor. Thanks to its simplicity, change of frame is done by changing angles. We choose the polar invariants of the elasticity tensor as a design variable.

The criterion in structural optimization may be for instance eigenfrequency, buckling, or compliance. In this work, the stiffness of the structure which is measured by the external work (compliance) is maximized. The optimization problem, which is based on variational methods similar to those that are used in continuum mechanics [Boutin et al. 2017; Andreaus et al. 2016], is equivalent to minimizing the compliance. Convex approximation methods such as MMA and GCMMA ((globally convergent) method of moving asymptotes) [Svanberg 1987; Zillober 1993] and descent algorithm methods such as SQP (sequential quadratic 
programming) [Arora and Belegundu 1984; Schittkowski 1985] and IPOPT (interior point optimizer) [Wächter and Biegler 2006] need the evaluation of the objective function as well as its gradient. The optimality criteria method computes the optimal values of design variables by expressing optimality conditions. Therefore, the optimality criteria method is less expensive than the methods above in term of numerical cost. This is the reason why a method similar to optimality criteria is used in this work. The algorithm used to solve the numerical problem is the alternate directions algorithm [Allaire and Kohn 1993]. One iterates between local minimizations with respect to the design variables and global minimizations corresponding to finite element calculations. Numerical results show the effectiveness of the method.

\section{Problem formulation:}

\section{simultaneous optimization of the material density and anisotropy}

Parametrization of the distributed material density and anisotropy. The shape of the structure is parametrized by a density field variable $\rho(x)$. This density variable defines at each point $x$ of the structure whether there is a material $(\rho(x)=1)$ or a void $(\rho(x)=0)$. Here $\rho(x)$ takes any value in $\left[\rho_{\min }, 1\right]$ while, in order to avoid singularity of the elasticity tensors, the lowest admissible value $\rho_{\min }$ is generally set to $10^{-3}$. Allowing $\rho(x)$ to be valued in the interval $\left[\rho_{\min }, 1\right]$ involves intermediate densities appearing in the optimum topologies. These intermediate densities involve gray areas that are difficult to interpret because they correspond to a mixture of void and material. To suppress gray areas, the density $\rho(x)$ is forced to tend to either $\rho_{\min }$ or 1 . The so-called SIMP method (solid isotropic material with penalization) [Bendsøe 1989] is used. This method uses an exponent $p \geq 2$ in order to interpolate the density $\rho(x)$. Optimized stiffness tensor $\underline{\underline{C}}(x)$ and compliance tensor $\underline{\underline{S}}(x)$ are expressed as functions of the considered material stiffness tensor $\underline{\underline{C_{0}}}(x)$ and compliance tensor $\underline{\underline{S_{0}}}(x)$ :

$$
\underline{\underline{C}}(x)=\rho(x)^{p} \underline{\underline{C_{0}}} \quad \Longleftrightarrow \quad \underline{\underline{S}}(x)=\frac{1}{\rho(x)^{p}} \underline{\underline{S_{0}}} .
$$

The elasticity tensor defines the stiffness properties of the anisotropic material. In the present work, spatial variations of the material anisotropy are allowed. A parametrization that allows one to express the elasticity tensor in a general frame in a simple way is necessary. Change of frame is cumbersome using the Cartesian representation. The polar method permits one to write the elasticity tensor with its intrinsic properties using tensor invariants. By doing so, changing frame becomes simple as one needs only to rotate an angle with respect to the frame. Thus, we choose to express the stiffness tensor with its polar invariants for an orthotropic 
material under assumption of plane stress. As the out-of-plane terms of the stress tensor vanish, the relation between the stress tensor and the strain tensor in the considered plane can be expressed only with the in-plane terms by introducing the reduced stiffness tensor $\underline{Q}$. Equations 2-2 show the relation between the polar components ( $T_{0}, T_{1}, R_{0}, R_{1}, \Phi_{0}$, and $\left.\Phi_{1}\right)$ and the Cartesian ones of the reduced stiffness tensor $\underline{Q}$ [Julien 2010; Vincenti and Desmorat 2011]:

$$
\begin{array}{lr}
Q_{1111}= & T_{0}+2 T_{1}+R_{0} \cos 4 \Phi_{0}+4 R_{1} \cos 2 \Phi_{1}, \\
Q_{1122}= & -T_{0}+2 T_{1}-R_{0} \cos 4 \Phi_{0}, \\
Q_{1112}= & R_{0} \sin 4 \Phi_{0}+2 R_{1} \sin 2 \Phi_{1}, \\
Q_{2222}= & T_{0}+2 T_{1}+R_{0} \cos 4 \Phi_{0}-4 R_{1} \cos 2 \Phi_{1}, \\
Q_{2212}= & -R_{0} \sin 4 \Phi_{0}+2 R_{1} \sin 2 \Phi_{1}, \\
Q_{1212}= & T_{0}-R_{0} \cos \Phi_{0} .
\end{array}
$$

Each Cartesian component of the reduced stiffness tensor is expressed with isotropic terms $T_{0}, T_{1}$ that do not depend on the orientation of the material and anisotropic terms $R_{0} e^{4 i \Phi_{0}}, R_{1} e^{2 i \Phi_{1}}$ that depend on the orientations $\Phi_{0}, \Phi_{1}$ of the material. The change of frame is done by changing these angles. The polar invariants are the moduli $T_{0}, T_{1}, R_{0}, R_{1}$ and the angle $\Phi_{0}-\Phi_{1}$. The isotropic parts do not influence the anisotropy of the material; thus, $T_{0}, T_{1}$ are supposed to remain constant (in composite laminated plates made of identical unidirectional layers (with the same material and same thickness in each layer), the homogenized isotropic part $T_{0}, T_{1}$ of the laminate is equal to the isotropic part $T_{0}^{\mathrm{EL}}, T_{1}^{\mathrm{EL}}$ of the elementary layer [Jibawy et al. 2011]). The material optimization is performed with respect to the anisotropic parts $R_{0}, R_{1}, \Phi_{1}$.

Figure 1 shows the decomposition of the reduced stress tensor's first Cartesian component $Q_{1111}$ for a composite made of long and straight carbon fibers in an epoxy matrix $\left(E_{\mathrm{L}}=112 \mathrm{GPa}, E_{\mathrm{T}}=8.2 \mathrm{GPa}, G_{\mathrm{LT}}=4.5 \mathrm{GPa}\right.$, and $\left.\nu_{\mathrm{LT}}=0.3 \mathrm{GPa}\right)$. The stiffness is expressed as the sum of terms that do not depend on the material orientation, $T_{0}$ and $T_{1}$, and terms that depend on the material orientation, $R_{0}$ and $R_{1}$. The $R_{0}$ and $R_{1}$ terms can take negative values (dashed lines) due to the cosine function (see (2-2)) and are $\frac{\pi}{4}$ - and $\frac{\pi}{2}$-periodic, respectively. The material orientation is equal to $0^{\circ}$. The apparent stiffness $Q_{1111}$ is maximized at $0^{\circ}$ as the $R_{0}$ and $R_{1}$ terms are both positive in this direction. It is minimized at $45^{\circ}$ because the $R_{0}$ and $R_{1}$ are respectively negative and null. When $R_{1}$ vanishes, there are only $\frac{\pi}{4}$-periodic terms: the material is square symmetric.

\section{Optimization constraint: maximum volume and thermodynamical admissibility} of the material. The optimization constraints are written in terms of the total volume amount of the structure and of the anisotropic part of the polar invariants by 


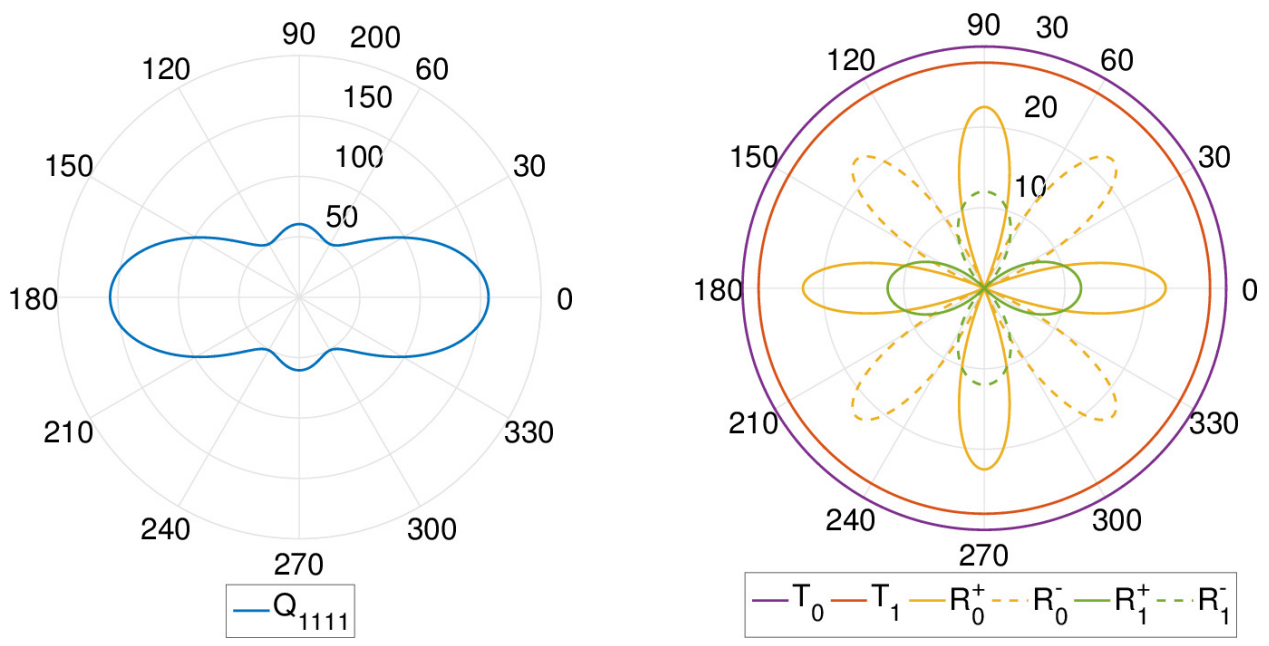

Figure 1. Left: representation of the first Cartesian component $Q_{1111}$ of the reduced stress tensor $Q$, in any orientation. Right: its decomposition into a sum of polar invariant terms $T_{0}, T_{1}, R_{0}, R_{1}$.

expressing their bounds. During the optimization, a target volume $V_{0}$ is defined for the structure. The volume $V$ is equal to the material density $\rho(x)$ integrated in the domain $\Omega$. At each step of the optimization, the volume must satisfy the optimization constraint

$$
V=\int_{\Omega} \rho(x) d x=V_{0} .
$$

The material to be designed is imposed to be orthotropic. For an orthotropic material,

$$
\Phi_{0}-\Phi_{1}=K \frac{\Pi}{4} \quad \text { with } K=0,1 .
$$

The orthotropic material used in this paper is taken to be as general as possible: the optimized orthotropic material is thermodynamically admissible, which means that the stiffness tensor is positive definite. The analytical bounds of the polar invariants are [Vannucci 2005]

$$
\left\{\begin{aligned}
T_{0} & >0, \\
T_{1} & >0, \\
T_{0} & >R_{0}, \\
T_{0} T_{1} & >R_{1}^{2}, \\
T_{1}\left(T_{0}^{2}-R_{0}^{2}\right) & >2 R_{1}^{2}\left(T_{0}-R_{0} \cos 4\left(\Phi_{0}-\Phi_{1}\right)\right) .
\end{aligned}\right.
$$

Double minimization of the complementary energy. In topology optimization, criteria such as buckling, frequency, or compliance may be considered; see for instance [Deaton and Grandhi 2014]. In this paper, we aim at maximizing the global 
structural stiffness measured by the compliance which is the external work. The criterion is written as

$$
\text { Criterion }=\int_{\Omega} \boldsymbol{f} \cdot \boldsymbol{u} d V+\int_{\Gamma_{1}} \boldsymbol{F} \cdot \boldsymbol{u} d S .
$$

The domain $\Omega$ is split into two boundaries: $\Gamma_{0}$ where a zero displacement is imposed and $\Gamma_{1}$ where a surface load $\boldsymbol{F}$ is applied. Then $\boldsymbol{f}$ is the volume load and $\boldsymbol{u}$ the displacement vector. The more the structure is rigid, the lower is the external work. Thus, maximizing the global structural stiffness means minimizing the compliance. Moreover, the compliance is equal to double the complementary energy. The optimization is made with respect to the density and the anisotropic part of the stiffness tensor polar invariants:

$$
\min _{\left\{\rho, R_{0}, R_{1}, \Phi_{1}\right\}} \int_{\Omega} \boldsymbol{f} \cdot \boldsymbol{u} d V+\int_{\Gamma_{1}} \boldsymbol{F} \cdot \boldsymbol{u} d S=\min _{\left\{\rho, R_{0}, R_{1}, \Phi_{1}\right\}} \int_{\Omega} \underline{\sigma}: \underline{\underline{C}}^{-1}: \underline{\sigma} d V
$$

The complementary energy theorem claims that the complementary energy can be written as the minimization of a positive quantity with respect to the statically admissible (SA) stress field $\underline{\tau}$ :

$$
\int_{\Omega} \underline{\sigma}: \underline{\underline{C}}^{-1}: \underline{\sigma} d V=\min _{\underline{\tau} \mathrm{SA}} \int_{\Omega} \underline{\tau}: \underline{\underline{C}}^{-1}: \underline{\tau} d V .
$$

The stress field $\underline{\tau}$ satisfies the elasticity problem $(\mathrm{P})$, with assumption of small strains and small displacements:

$$
\left\{\begin{aligned}
\operatorname{div} \underline{\tau}+\boldsymbol{f} & =0 & & \text { in } \Omega, \\
\underline{\tau} \cdot \boldsymbol{n} & =\boldsymbol{F} & & \text { on } \Gamma_{1}, \\
\underline{\tau} & =\underline{\underline{C}}: \underline{\epsilon}(\boldsymbol{u}) & & \text { in } \Omega, \\
\boldsymbol{u} & =0 & & \text { on } \Gamma_{0},
\end{aligned}\right.
$$

where $\underline{\epsilon}(\boldsymbol{u})=\frac{1}{2}\left(\underline{\nabla \boldsymbol{u}}+\underline{\nabla \boldsymbol{u}} \underline{i}^{T}\right)$ is the strain tensor. By replacing the expression of the complementary energy in (2-7), the optimization problem is written as a double minimization with respect to the design variables $\left\{\rho, R_{0}, R_{1}, \Phi_{1}\right\}$ and to the stress field $\underline{\tau}$. The density variable is subject to a maximal volume constraint, and the polar invariants of the stiffness tensor are constrained by thermodynamic bounds:

$$
\min _{\left\{\rho, R_{0}, R_{1}, \Phi_{1}\right\}} \min _{\underline{\tau} \mathrm{SA}} \int_{\Omega} \underline{\tau}: \underline{\underline{C}}^{-1}: \underline{\tau} d V
$$

with

$$
\left\{\begin{aligned}
\int_{\Omega} \rho(x) d x & =V_{0}, \\
T_{0} & >R_{0}, \\
T_{0} T_{1} & >R_{1}^{2}, \\
T_{1}\left(T_{0}^{2}-R_{0}^{2}\right) & >2 R_{1}^{2}\left(T_{0}-R_{0} \cos 4\left(\Phi_{0}-\Phi_{1}\right)\right), \\
\Phi_{0}-\Phi_{1} & =K(\Pi / 4), \quad K=0,1 .
\end{aligned}\right.
$$




\section{Complementary energy minimization using the alternate direction algorithm}

Local minimizations of the complementary energy. Since the design variables $\left\{\rho, R_{0}, R_{1}, \Phi_{1}\right\}$ are subject only to algebraic constraints, the minimization with respect to them can be put inside the integral:

$$
\min _{\underline{\tau} \mathrm{SA}} \int_{\Omega} \min _{\left\{\rho, R_{0}, R_{1}, \Phi_{1}\right\}} \underline{\tau}: \underline{\underline{C}}^{-1}: \underline{\tau} d V \quad \text { with }(\mathrm{C}) .
$$

The minimization of the complementary energy with respect to the design variables is solved locally in each point of the domain, for a fixed stress state. Since the density variable $\rho(x)$ and the anisotropy variables $\left\{R_{0}, R_{1}, \Phi_{1}\right\}$ are independent, the minimization is split into two steps. First the complementary energy is minimized with respect to the anisotropy variables, taking into account the algebraic constraints related to thermodynamic bounds. Second, the minimization with respect to the density variable is performed.

The complementary energy can be written as a simple function of the polar invariants of the stiffness tensor and the stress tensor. Calculating its derivative is then straightforward. Hence, the minimization of the complementary energy with respect to the anisotropy variables is done analytically. The optimal values of $\left\{R_{0}, R_{1}, \Phi_{1}\right\}$ depending on the stress field are given in [Julien 2010] and are shown in Table 1, introducing the ratio $R /|T|$ where $R$ and $T$ are the spherical and deviatoric parts of the stress tensor, respectively. The optimal orthotropic material orientation is in the same direction as the principal direction of the stress tensor with maximal absolute value. The optimal values of polar invariants $R_{0}$ and $R_{1}$ depend on the ratio $R /|T|$.

The volume constraint is taken into account in the minimization step with respect to the density variable $\rho(x)$ through the introduction of a Lagrangian multiplier $k$ :

$$
\min _{\rho} \frac{1}{\rho(x)^{p}} \underline{\tau}: \underline{\underline{C}}^{-1}\left(R_{0}^{\mathrm{opt}}, R_{1}^{\mathrm{opt}}, \Phi_{1}^{\mathrm{opt}}\right): \underline{\tau}+k \rho(x)
$$

\begin{tabular}{|c|c|c|c|}
\hline$X=R /|T|$ & 0 & $\sqrt{T_{0} /\left(2 T_{1}\right)}$ & $\sqrt{T_{0} / T_{1}}$ \\
\hline$\Phi_{1}^{\text {opt }}$ & \multicolumn{3}{|c|}{$\operatorname{Dir}\left\{\max \left(\left|\sigma_{\mathrm{I}}\right|,\left|\sigma_{\mathrm{II}}\right|\right)\right\}$} \\
\hline$R_{0}^{\text {opt }}$ & $0 \leq R_{0}^{\text {opt }}<T_{0}$ & $2 T_{1} X^{2}-T_{0}<R_{0}^{\text {opt }}<T_{0}$ & $T_{0}^{-}$ \\
\hline$R_{1}^{\text {opt }}$ & \multicolumn{2}{|c|}{$T_{1} X$} & $T_{0}^{-} / X$ \\
\hline
\end{tabular}

Table 1. Optimal values [Julien 2010, Table 3.8] of the polar invariants $\left\{R_{0}, R_{1}, \Phi_{1}\right\}$ depending on the stress field, in the case $\Phi_{0}-\Phi_{1}=K \frac{\pi}{4}=0(K=0)$. 
The minimum of the local energy is attained by setting to zero the variation of (3-2) with respect to the density field, which yields

$$
\rho(x)=\left(\frac{p \underline{\tau}: \underline{C}^{-1}\left(R_{0}^{\mathrm{opt}}, R_{1}^{\mathrm{opt}}, \Phi_{1}^{\mathrm{opt}}\right): \underline{\tau}}{k}\right)^{1 /(p+1)} .
$$

The value of $k$ is calculated so that the volume constraint is satisfied.

Optimization algorithm. The double minimization is solved with a fixed point method by considering the optimality conditions. At each iteration of the optimization, the minimization with respect to the design variables $\left\{\rho, R_{0}, R_{1}, \Phi_{1}\right\}$ is first performed; then the minimization with respect to the stress field $\underline{\tau}$ is operated. The minimization with respect to the stress field $\underline{\tau}$ corresponds to a finite element analysis thanks to the complementary energy theorem. The minimizations are treated alternatively and separately. This method is an extension of the alternate direction algorithm introduced in [Allaire and Kohn 1993]. Thanks to the polar method, the local complementary energy is written with simple expressions. Hence, the local minimizations are solved analytically.

The advantage of the alternate direction algorithm is its simplicity and low numerical cost as the method iterates between local minimizations solved analytically and finite element calculations of stresses. The work in [Desmorat 2013] shows also the convergence of the algorithm for a compliance minimization problem. The cost of the algorithm is directly related to the finite element calculation cost. Finally, the algorithm can take into account a large number of variables.

\section{Numerical results}

Numerical results are presented in this section to prove the efficiency of the method. The optimization is made for a two-dimensional orthotropic linear elastic material. A support beam from a civil aircraft produced by Messerschmidt-Bölkow-Blohm, called the MBB beam, is optimized here. The beam carries the floor in the fuselage of an Airbus airliner. Maximizing its rigidity has become a classical problem in topology optimization (see [Zhou and Rozvany 1991] for example). The design domain is a rectangle clamped with respect to the $x$-axis at the left side and with respect to the $y$-axis at the bottom of the right side (orange-colored dot in Figure 2). A load is applied on the top of the left side. The domain size is $40 \mathrm{~mm} \times 20 \mathrm{~mm}$ discretized with a rectangular $80 \times 40$ mesh. The volume constraint is fixed at $50 \%$ of the total volume. The initial density is set to 1 in every element of the mesh. The initial material is an isotropic material where the values of $T_{0}$ and $T_{1}$ correspond to the isotropic part of a monolayered composite made of long and straight carbon fibers in an epoxy matrix: $T_{0}=26.88 \mathrm{GPa}$ and $T_{1}=24.74 \mathrm{GPa}$. 


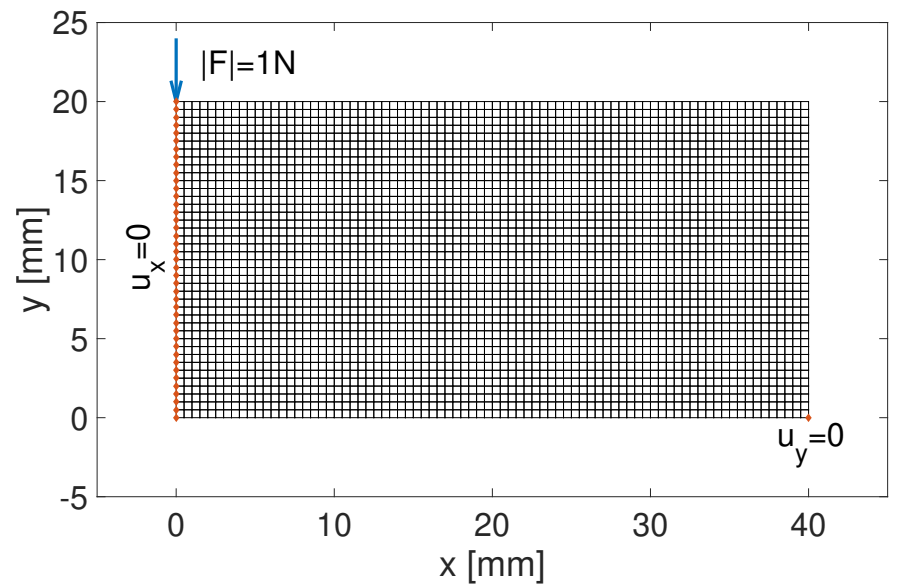

Figure 2. Boundary conditions for the MBB beam problem.

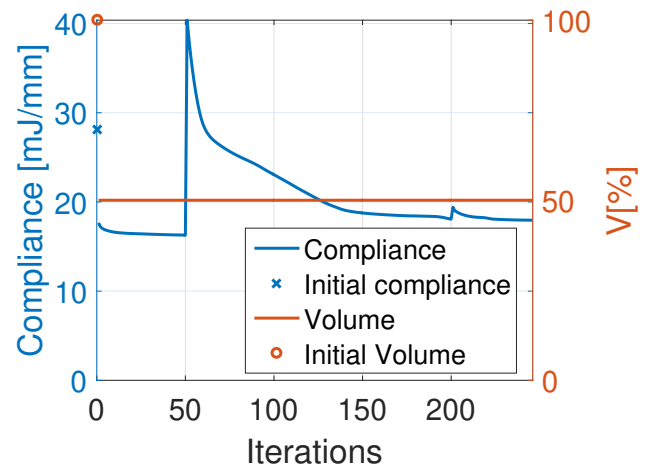

Figure 3. Compliance and volume with respect to optimization iterations.

Convergence. The compliance and the volume are displayed as functions of the iterations in Figure 3. The strategy of penalizing the density is made in three steps during which the exponent $p$ in $\rho(x)^{p}$ is increased gradually. First, the convex problem corresponding to $p=1$ is treated. The convexity of the problem when taking $p=1$ is proved theoretically in [Allaire et al. 1997]. This means that the solution at the end of the iterations where $p=1$ is a global minimum, making the solution independent of the initialization. Second, starting from this global minimum point, the solution is forced to be a $0 / 1$ layout by increasing $p$ to 3 . Finally, $p$ is taken to be equal to 5 to suppress definitely intermediate density.

Except for the first iteration, the volume does not change through the iterations as it is constrained here to be equal to $50 \%$ of the total feasible volume. The compliance decreases at each of three steps $(p=1, p=3$, and $p=5)$. At each step, convergence is reached when the variation of compliance between two consecutive 


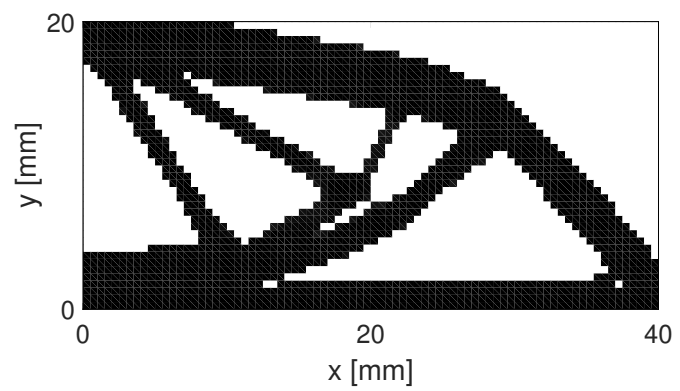

Figure 4. Optimal topology of the MBB beam with $50 \%$ volume amount.

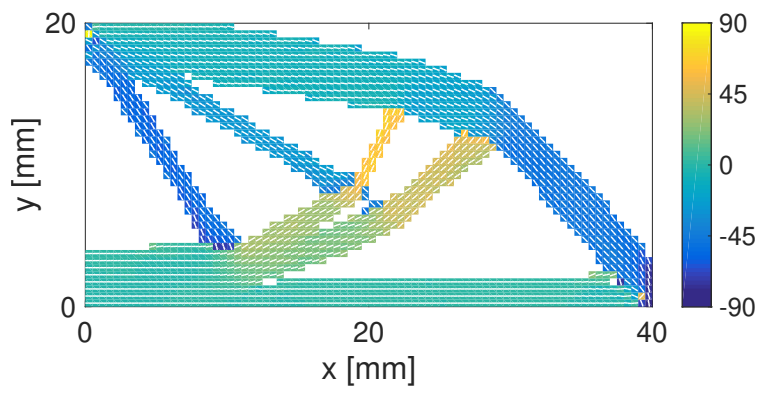

Figure 5. Optimal distribution of orthotropy direction.

iterations is less than $0.1 \%$ and the variation of the local densities is less than $0.01 \%$. The compliance increases when the value of $p$ is increased because the structure becomes suddenly less stiff when the interpolation of intermediate density values is changed. We can observe that, at the end of the optimization, the compliance has converged.

Optimal distribution of density. Figure 4 shows the optimal shape of the structure where black represents the presence of material and white its absence. The material is pictured when the density value is above 0.9 . To avoid numerical problems such as checkerboard, a filter is used: the density of an element depends on the density of its neighbors so that there is no sharp discontinuity of the density in the structure. The neighbor elements that influence the considered element are defined by a radius filter. The filtering method used in this work is similar to the method of filtering sensitivities [Bendsøe and Sigmund 2003]. A structured mesh is used in this work. The filter radius permits us to suppress the checkerboard problem. For a given value of the radius, it has been observed numerically that the mesh dependency of the optimal topology seems to vanish when the element size is small enough compared to the filter radius. However, the optimal shape depends on values of the radius filter, which can be interpreted as a minimal bar width. 

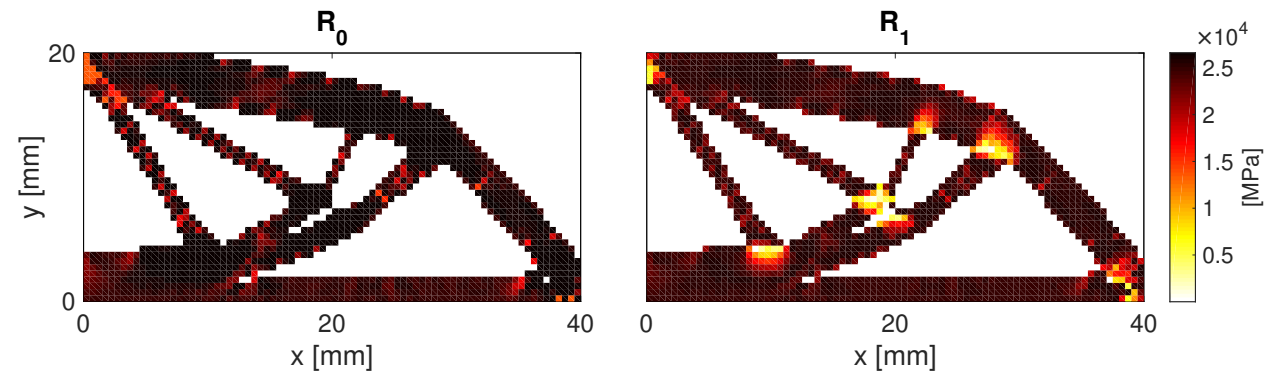

Figure 6. Optimal distribution of the stiffness tensor anisotropic polar invariants $R_{0}$ (left) and $R_{1}$ (right).

Optimal distribution of orthotropy. In the optimal shape, the orthotropy is distributed: the material orthotropy changes continuously inside the structure. The optimal orthotropy direction $\Phi_{1}^{\text {opt }}$ is presented in Figure 5. It is aligned with the stress principal direction. The direction changes continuously throughout the structure as the stress field is continuous, except on the areas that are solicited in shearing, where a bar intersect another one. In these areas, the optimized material is square symmetric (i.e., $R_{1}=0$ ). The apparent stiffness, having the same value in $\Phi_{1}$ modulo $\pi / 4$, is continuous in space in the optimal design. We illustrate the distribution of the moduli $R_{0}$ and $R_{1}$ in Figure 6 . The $R_{0}$ values are set to be quasiconstant whereas the $R_{1}$ values vary from 0 to $25 \mathrm{GPa}$. We can see that in the areas where $R_{1}$ are minimum, the shearing is maximum. The optimal materials in these areas where $R_{1}=0$ are square symmetric materials, stiffened in two orthogonal directions. When $R_{1}$ is maximum, the optimal material is stiffened in one direction because it is solicited mostly in traction or compression.

\section{Conclusion}

The proposed methodology presented in this paper concurrently gives the spatial material distribution and the material anisotropy distribution by minimizing the compliance. The optimization strategy is based on an optimality criteria method in which one iterates successively and separately between local minimizations and finite element calculations. In order to avoid mesh size dependency, it could be of interest to develop such an optimization procedure with the use of some generalized continuum theories. Parametrizing the shape of the structure with a density variable and the anisotropy of the material with polar invariants allows for solving the local minimizations analytically. Thus, the computational cost of the algorithm corresponds to the finite element calculations. The method is straightforward to implement and gives coherent results from a mechanical viewpoint. Indeed, the optimal material where the structure is loaded in shear is square symmetric, because 
it has to be stiffened in two orthogonal directions. Areas loaded in traction or compression have an optimal material stiffened in one direction only. The presented optimization methodology is very promising when considering real composite material distribution, as the only change to be performed will be on the admissible set of polar parameters that should take into account the feasibility of the considered composite material.

\section{References}

[Allaire and Delgado 2016] G. Allaire and G. Delgado, "Stacking sequence and shape optimization of laminated composite plates via a level-set method", J. Mech. Phys. Solids 97 (2016), 168-196.

[Allaire and Kohn 1993] G. Allaire and R. V. Kohn, "Optimal design for minimum weight and compliance in plane stress using extremal microstructures", European J. Mech. A Solids 12:6 (1993), 839-878.

[Allaire et al. 1997] G. Allaire, E. Bonnetier, G. Francfort, and F. Jouve, "Shape optimization by the homogenization method", Numer. Math. 76:1 (1997), 27-68.

[Andreaus et al. 2016] U. Andreaus, F. dell'Isola, I. Giorgio, L. Placidi, T. Lekszycki, and N. L. Rizzi, "Numerical simulations of classical problems in two-dimensional (non) linear second gradient elasticity", Internat. J. Engrg. Sci. 108 (2016), 34-50.

[Arora and Belegundu 1984] J. S. Arora and A. D. Belegundu, "Structural optimization by mathematical programming methods", AIAA J. 22:6 (1984), 854-856.

[Bendsøe 1989] M. P. Bendsøe, “Optimal shape design as a material distribution problem”, Struct. Opt. 1:4 (1989), 193-202.

[Bendsøe and Sigmund 2003] M. P. Bendsøe and O. Sigmund, Topology optimization: theory, methods and applications, Springer, 2003.

[Boutin et al. 2017] C. Boutin, F. dell'Isola, I. Giorgio, and L. Placidi, "Linear pantographic sheets: asymptotic micro-macro models identification”, Math. Mech. Complex Syst. 5:2 (2017), 127-162.

[Deaton and Grandhi 2014] J. D. Deaton and R. V. Grandhi, "A survey of structural and multidisciplinary continuum topology optimization: post 2000”, Struct. Multidiscip. Optim. 49:1 (2014), $1-38$.

[Desmorat 2013] B. Desmorat, "Structural rigidity optimization with an initial design dependent stress field: application to thermo-elastic stress loads", Eur. J. Mech. A Solids 37 (2013), 150-159.

[Ghiasi et al. 2009] H. Ghiasi, D. Pasini, and L. Lessard, "Optimum stacking sequence design of composite materials, I: Constant stiffness design”, Compos. Struct. 90:1 (2009), 1-11.

[Ghiasi et al. 2010] H. Ghiasi, K. Fayazbakhsh, D. Pasini, and L. Lessard, "Optimum stacking sequence design of composite materials, II: Variable stiffness design”, Compos. Struct. 93:1 (2010), $1-13$.

[Irisarri et al. 2009] F.-X. Irisarri, D. H. Bassir, N. Carrere, and J.-F. Maire, "Multiobjective stacking sequence optimization for laminated composite structures”, Compos. Sci. Tech. 69:7-8 (2009), 983990.

[Jibawy et al. 2011] A. Jibawy, C. Julien, B. Desmorat, A. Vincenti, and F. Léné, "Hierarchical structural optimization of laminated plates using polar representation”, Int. J. Solids Struct. 48:18 (2011), 2576-2584.

[Julien 2010] C. Julien, Conception optimale de l'anisotropie dans les structures stratifiées à rigidité variable par la méthode polaire-génétique, Ph.D. thesis, UPMC, 2010. 
[Peeters et al. 2015] D. Peeters, D. van Baalen, and M. Abdallah, "Combining topology and lamination parameter optimisation", Struct. Multidiscip. Optim. 52:1 (2015), 105-120.

[Rion and Bruyneel 2007] V. Rion and M. Bruyneel, "Topology optimization of membranes made of orthotropic material", pp. 107-120 in Collection of papers from Professor Nguyen Dang Hung's former students, edited by G. DeSaxcé and N. Moës, Vietnam National University, 2007.

[Rojas-Labanda and Stolpe 2015] S. Rojas-Labanda and M. Stolpe, "Benchmarking optimization solvers for structural topology optimization”, Struct. Multidiscip. Optim. 52:3 (2015), 527-547.

[Schittkowski 1985] K. Schittkowski, "Software for mathematical programming”, pp. 383-451 in Computational mathematical programming (Bad Windsheim, Germany, 1984), edited by K. Schittkowski, NATO Adv. Sci. Inst. Ser. F Comput. Systems Sci. 15, Springer, 1985.

[Sigmund and Maute 2013] O. Sigmund and K. Maute, "Topology optimization approaches", Struct. Multidiscip. Optim. 48:6 (2013), 1031-1055.

[Sørensen and Lund 2013] S. N. Sørensen and E. Lund, "Topology and thickness optimization of laminated composites including manufacturing constraints", Struct. Multidiscip. Optim. 48:2 (2013), 249-265.

[Svanberg 1987] K. Svanberg, "The method of moving asymptotes-a new method for structural optimization”, Internat. J. Numer. Methods Engrg. 24:2 (1987), 359-373.

[Vannucci 2005] P. Vannucci, "Plane anisotropy by the polar method", Meccanica 40:4-6 (2005), 437-454.

[Verchery 1982] G. Verchery, "Les invariants des tenseurs d'ordre 4 du type de l'élasticité", pp. 93104 in Comportment méchanique des solides anisotropes (Villard-de-Lans, France, 1979), edited by J.-P. Boehler, Colloques Int. Centre Nat. Recherche Sci. 295, Springer, 1982.

[Vincenti and Desmorat 2011] A. Vincenti and B. Desmorat, "Optimal orthotropy for minimum elastic energy by the polar method", J. Elasticity 102:1 (2011), 55-78.

[Wächter and Biegler 2006] A. Wächter and L. T. Biegler, "On the implementation of an interiorpoint filter line-search algorithm for large-scale nonlinear programming", Math. Program. 106:1, Ser. A (2006), 25-57.

[Zhou and Rozvany 1991] M. Zhou and G. I. N. Rozvany, "The COC algorithm, II: Topological, geometrical and generalized shape optimization”, Comp. Meth. Appl. Mech. Eng. 89:1-3 (1991), 309-336.

[Zillober 1993] C. Zillober, "A globally convergent version of the method of moving asymptotes", Struct. Opt. 6:3 (1993), 166-174.

Received 12 Oct 2017. Revised 7 May 2018. Accepted 14 Jun 2018.

NARINDRA RANAIVOMIARANA: narindra.ranaivomiarana@onera.fr

Centre de Châtillon, Office National d'Etudes et de Recherches Aérospatiales, Chatillon, France

FRANÇOIS-XAVIER IRISARRI: francois-xavier.irisarri@onera.fr

Centre de Châtillon, Office National d'Etudes et de Recherches Aérospatiales, Chatillon, France

DIMITRI BETTEBGHOR: dimitri.bettebghor@onera.fr

Centre de Châtillon, Office National d'Etudes et de Recherches Aérospatiales, Chatillon, France

BORIS DESMORAT: boris.desmorat@sorbonne-universite.fr

Institut Jean Le Rond d'Alembert, Sorbonne Université, Paris, France 
EDITORIAL BOARD

ANTONIO CARCATERRA

ERIC A. CARLEN

FRANCESCO DELL'ISOLA

RAFFAELE ESPOSITO

ALBERT FANNJIANG

Gilles A. FrancFort

PieRANGelo MARCATI

JEAN-JACQUES MARIGO

PETER A. MARKOWICH

MARTIN OSTOJA-STARZEWSKI

PIERRE SEPPECHER

DAVID J. STEIGMANN

PAUl STEINMANN

PierRe M. SuQueT

MANAGING EDITORS

MICOL AMAR

CORRADO LATTANZIO

ANGELA MADEO

MARTIN OSTOJA-STARZEWSKI

ADVISORY BOARD

ADNAN AKAY

Holm AltenbaCH

MICOL AMAR

HARM ASKES

TEODOR ATANACKOVIĆ

VICTOR BERDICHEVSKY

GUY BOUCHITTÉ

ANDREA BRAIDES

ROBERTO CAMASSA

MAURO CARFORE

ERIC DARVE

FELIX DARVE

ANNA DE MASI

GianPiETRo DEL PIERo

EMmANUELE Di BENEDETTO

BERNOLD FIEDLER

IRENE M. GAMBA

DAVID Y. GAO

SERGEY GAVRILYUK

Timothy J. HEALEY

DOMINIQUE JEULIN

ROGER E. KHAYAT

CORRADO LATTANZIO

ROBERT P. LIPTON

ANGELO LUONGO

ANGELA MADEO

JUAN J. MANFREDI

CARlo MARCHIORO

ROBERTO NATALINI

PATRIZIO NEFF

ANDREY PIATNITSKI

ERRICO PRESUTtI

MARIO PULVIRENTI

LuCiO RuSSO

Miguel A. F. SANJUAN

PATRICK SElVAdURAI

ALEXANDER P. SEYRANIAN

MiroslaV ŠILHAVÝ

GUIDO SWEERS

ANTOINETTE TORDESILLAS

LEV TRUSKINOVSKY

JUAN J. L. VELÁZQUEZ

VINCENZO VESPRI

ANGELO VULPIANI msp.org/memocs

Università di Roma "La Sapienza", Italia

Rutgers University, USA

(CO-CHAIR) Università di Roma "La Sapienza", Italia

(TREASURER) Università dell'Aquila, Italia

University of California at Davis, USA

(CO-CHAIR) Université Paris-Nord, France

Università dell' Aquila, Italy

École Polytechnique, France

DAMTP Cambridge, UK, and University of Vienna, Austria

(CHAIR MANAGING EDITOR) Univ. of Illinois at Urbana-Champaign, USA

Université du Sud Toulon-Var, France

University of California at Berkeley, USA

Universität Erlangen-Nürnberg, Germany

LMA CNRS Marseille, France

Università di Roma "La Sapienza", Italia

Università dell' Aquila, Italy

Université de Lyon-INSA (Institut National des Sciences Appliquées), France

(CHAIR MANAGING EDITOR) Univ. of Illinois at Urbana-Champaign, USA

Carnegie Mellon University, USA, and Bilkent University, Turkey

Otto-von-Guericke-Universität Magdeburg, Germany

Università di Roma "La Sapienza", Italia

University of Sheffield, UK

University of Novi Sad, Serbia

Wayne State University, USA

Université du Sud Toulon-Var, France

Università di Roma Tor Vergata, Italia

University of North Carolina at Chapel Hill, USA

Università di Pavia, Italia

Stanford University, USA

Institut Polytechnique de Grenoble, France

Università dell'Aquila, Italia

Università di Ferrara and International Research Center MEMOCS, Italia

Vanderbilt University, USA

Freie Universität Berlin, Germany

University of Texas at Austin, USA

Federation University and Australian National University, Australia

Université Aix-Marseille, France

Cornell University, USA

École des Mines, France

University of Western Ontario, Canada

Università dell' Aquila, Italy

Louisiana State University, USA

Università dell'Aquila, Italia

Université de Lyon-INSA (Institut National des Sciences Appliquées), France University of Pittsburgh, USA

Università di Roma "La Sapienza", Italia

Istituto per le Applicazioni del Calcolo "M. Picone", Italy

Universität Duisburg-Essen, Germany

Narvik University College, Norway, Russia

Università di Roma Tor Vergata, Italy

Università di Roma "La Sapienza", Italia

Università di Roma "Tor Vergata”, Italia

Universidad Rey Juan Carlos, Madrid, Spain

McGill University, Canada

Moscow State Lomonosov University, Russia

Academy of Sciences of the Czech Republic

Universität zu Köln, Germany

University of Melbourne, Australia

École Polytechnique, France

Bonn University, Germany

Università di Firenze, Italia

Università di Roma La Sapienza, Italia

MEMOCS (ISSN 2325-3444 electronic, 2326-7186 printed) is a journal of the International Research Center for the Mathematics and Mechanics of Complex Systems at the Università dell'Aquila, Italy.

Cover image: "Tangle" by @ John Horigan; produced using the Context Free program (contextfreeart.org).

\section{PUBLISHED BY}

mathematical sciences publishers nonprofit scientific publishing

http://msp.org/

(C) 2018 Mathematical Sciences Publishers 
Mathematics and Mechanics of Complex Systems vol. 6 no. 4

A model for interfaces and its mesoscopic limit

Michele Aleandri and Venanzio Di Giulio

Optimal orthotropy and density distribution of two-dimensional structures

Narindra Ranaivomiarana, François-Xavier Irisarri, Dimitri

Bettebghor and Boris Desmorat

A multiphysics stimulus for continuum mechanics bone remodeling 307

Daniel George, Rachele Allena and Yves Rémond

On linear non-local thermo-viscoelastic waves in fluids

Joe D. Goddard

Heterogeneous directions of orthotropy in three-dimensional

structures: finite element description based on diffusion equations

Rachele Allena and Christophe Cluzel

A general method for the determination of the local orthotropic directions of heterogeneous materials: application to bone structures using $\mu \mathrm{CT}$ images

Christophe Cluzel and Rachele Allena

MEMOCS is a journal of the International Research Center for the Mathematics and Mechanics of Complex Systems at the Università dell' Aquila, Italy.

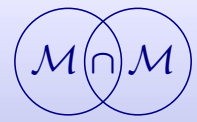

